\title{
Neue Entwicklungen bei Impfstoffen für Kinder und Jugendliche
}

Aktuelle neue Impfstoff-Entwicklungen für das Kindes- und Jugendalter betreffen einerseits Anpassungen und/oder Optimierungen vorhandener Impfstoffe und Impfkonzepte oder adressieren bislang nicht befriedigend gelöste Fragestellungen in der Prävention von Infektionskrankheiten.

\section{Neuer Meningokokken Typ B-Impfstoff}

Seit 2017 ist ein neuer, aus zwei Komponenten bestehender MenB-Impfstoff (rLP2086-MenB-FHbp) durch die EMA zugelassen [1]. Der Impfstoff rLP2086 besteht aus zwei rekombinant hergestellten Varianten des konservierten bakteriellen Oberflächenmoleküls Human-Faktor-HBindungsprotein (FHbp, auch bekannt als LP2086). FHbp ist ein Antigen, das von mehr als 97\% der MenB-Stämme exprimiert wird. Bakterien binden mittels FHbp das Regulatorprotein $\mathrm{H}$, das die Komplement-vermittelte Lyse hemmt. Durch die Bindung von Protein $\mathrm{H}$ blockiert das Bakterium aktiv Bindung und Aktivierung der lytischen Kaskade des Komplementsystems. Der Impfstoff rLP2086 induziert die Produktion von Antikörpern gegen FHbp, die spezifisch diesen Rezeptor besetzen und die Bindung von Protein $\mathrm{H}$ verhindern sowie gleichzeitig an dieser Position eine komplementvermittelte Lysierung der Bakterienzellwand einleiten. Aufgrund des stärkeren Nebenwirkungsprofils bei jungen Kindern ist der Impfstoff in Deutschland erst ab einem Alter von zehn Jahren zugelassen. In den USA wurde die Zulassung vor wenigen Wochen um das Altersfenster zwischen 2 und 10 Jahren erweitert.

Eine im Dezember 2017 im The New England Journal of Medicine veröffentlichte Arbeit [2] berichtet über zwei Phase-IIIStudien mit Jugendlichen (10-18 Jahre) und jungen Erwachsenen (18-25 Jahre), die für beide Altersgruppen eine gute Immunogenität und Reaktogenität ergaben.

In einer Studie wurde der Impfstoff mit 3596 gesunden Kindern und Jugendlichen zwischen 10 und 18 Jahren geprüft. Die ju- gendlichen Teilnehmer erhielten randomisiert entweder den MenB-Impfstoff (Schema 0, 2, 3 Monate) oder $\mathrm{NaCl}$-Lösung/ HAV-Impfstoff in der Kontrollgruppe. In der zweiten Studie erhielten 3304 gesunde Erwachsene zwischen 18 und 25 Jahren randomisiert $(2: 1)$ den Kandidat-Impfstoff (Schema 0, 2, 4 Monate) oder physiologische Kochsalzlösung als Kontrolle. Primärer Endpunkt der Studien war ein Anstieg des Antikörper-Titers gegen die FHbp-Proteine der vier primären Meningokokken-B-Subtypen (insgesamt gibt es 13 Subtypen) um mindestens das Vierfache. Bei den Jugendlichen zeigte sich bereits nach der zweiten Impfung ein ausreichender Antikörpertiter-Anstieg bei 56 bis 85,3\% der Probanden. Nach der dritten Impfung stieg dieser Anteil auf 78,8 bis 90,2\%. Unter den jungen Erwachsenen hatten nach zwei Impfungen 54,6 bis $86,6 \%$ einen ausreichenden Impfschutz und nach drei Impfungen 78,9 bis $89,7 \%$. Nach der dritten Dosierung erreichten $83 \%$ der Jugendlichen und $84 \%$ der $\mathrm{Er}$ wachsenen einen ausreichenden Impfschutz gegen alle vier primären Stämme.

\section{Quadrivalente inaktivierte Influenza-Impfstoffe für Säuglinge und Kleinkinder}

Nachdem die WHO schon 2013 auf die Vorteile von quadrivalenten InfluenzaImpfstoffen besonders im Hinblick auf die bessere Virus-Typen-Abdeckung hingewiesen hatte, haben verschiedene europäische Länder die Umstellung auf viervalente Impfstoffe vollzogen. Im Januar 2018 hat die STIKO die präferentielle Nutzung quadrivalenter Influenza-Impfstoffe (IIV4) in Deutschland empfohlen [3].

Während für ältere Kinder und Erwachsene quadrivalente Influenza-Vakzinen schon zugelassen sind, sind diese bisher für Säuglinge nicht verfügbar gewesen.

Eine randomisierte, verblindete, multinationale Phase-III-Studie mit 12018 gesunden Kindern im Alter von 6-35 Monaten über 5 Grippesaisons ergab nach zweima- liger Impfung mit einem quadrivalenten Impfstoff bei über $90 \%$ eine Influenza-spezifische Antikörper-Antwort [4]. Vergleichbare Resultate wurden mit einem quadrivalenten Impfstoff eines weiteren europäischen Herstellers erzielt. In der randomisierten, verblindeten, multizentrischen Phase-III-Studie wurde die Immunogenität und Sicherheit von IIV4 bei Säuglingen und Kleinkindern im Alter von 6 bis 35 Monaten untersucht [5]. In die mehrjährige Studie wurden insgesamt 5806 Kinder aufgenommen, die entweder die quadrivalente Kandidatvakzine (QIV - n: 2715) oder einen kommerziellen trivalenten Influenza-Impfstoff (TIV - n: 369) oder eine Placebo-Impfung (n: 2722) erhielten. Die Wirksamkeit gegenüber RT-PCR-bestätigter Influenza betrug 52\% gegenüber jedem Virus-Stamm, und $69 \%$ gegenüber den im Impfstoff enthaltenen Typen. Das Sicherheitsprofil von QIV war mit dem des zugelassenen TIV vergleichbar.

Die quadrivalenten Influenza-Totimpfstoffe zeigen eine gute Wirksamkeit von etwa $60 \%$ in der untersuchten Altersgruppe. Beide Produkte weisen ein mit trivalenten Impfstoffen vergleichbares Sicherheitsprofil auf und können ab der Saison 2018/ 2019 alternativ zu dem schon zugelassenen quadrivalenten, lebend-attenuierten Impfstoff (dieser erst ab dem 2. Lebensjahr) eingesetzt werden.

\section{Neuer Hepatitis-B-Impfstoff}

In den USA wurde in den vergangenen zehn Jahren ein neuer Hepatitis-B-Impfstoff (HBV) („HBsAg-1018“) entwickelt und 2018 als HEPLISAV-B ${ }^{\text {TM }}$ eingeführt. Es handelt sich um den einzigen Impfstoff, der mit einem Zwei-Dosis-Schema (4 Wochen Impfabstand) zur Prävention von Infektionen durch alle bekannten HBV-Subtypen bei Erwachsenen ab 18 Jahren verimpft werden kann. Etablierte HBV-Impfstoffe erfordern drei Impfungen, in der Regel über einen Sechs-Monate-Zeitraum.

HBsAg-1018 enthält als spezifisches Impfantigen $20 \mu \mathrm{g}$ rekombinantes $\mathrm{HBsAg}$ und ist mit einem Toll-like Rezeptor 9 (TLR9) 
Agonisten adjuvantiert. Das Molekül 1018 ist ein synthetisches Oligonukleotid mit immunstimulatorischen CpG-Motiven, die die angeborene Immunantwort gegenüber bakterieller/viraler DNA über TLR9 stimulieren und zu einer ausgeprägten Verstärkung der B- und T-zellulären Immunantwort führen.

Zwei Impfdosen innerhalb von 4 Wochen induzierten in klinischen Studien mit über 10000 Teilnehmern signifikant höhere Seroprotektionsraten als drei Dosen (über 24 Wochen) eines zugelassenen Aluminiumadjuvantierten Impfstoffes. Insbesondere bei Individuen mit chronischen Begleiterkrankungen und bekannter reduzierter HBV-Impfantwort scheint der Impfstoff den bisher verfügbaren Vakzinen überlegen zu sein. Die verstärkte Immunantwort aufgrund des als Adjuvans eingesetzten TLR-9 Agonisten geht allerdings mit einer ebenfalls verstärkten Reaktogenität (lokal und systemisch) einher, die sich jedoch nicht signifikant von den zugelassenen Impfstoffen unterscheidet.

\section{Neuer Pertussis-Impfstoff}

Epidemiologische Untersuchungen der vergangenen Jahre zeigen, dass der Schutz nach Impfung mit azellulären PertussisImpfstoffen schneller schwindet als die erworbene Immunität nach überstandener Infektion oder nach Impfung mit Ganzzell-Impfstoffen. So hat die WHO empfohlen, den Wechsel von Ganzzell- zu azellulären Pertussis-Impfstoffen zur primären Immunisierung von Säuglingen nur dann in Betracht zu ziehen, wenn zusätzliche regelmäßige Booster und/oder mütterliche Immunisierung in den nationalen Impfprogrammen gewährleistet und erhalten werden können. In Ländern mit hoher Durchimpfungsrate für azelluläre Pertussis-Impfstoffe wird derzeit von einer Schutzdauer von etwa 5-6 Jahren ausgegangen. Diese Entwicklung macht zukünftig die Anpassung von Impfkonzepten und/oder die Entwicklung neuer Pertussis-Impfstoffe erforderlich.

Sricharoenchai et al. [6] berichten in mehreren Publikationen über die Entwicklung eines neuen Pertussis-Impfstoffs in Thailand, der in Studien eine bessere Immuno- genität und Persistenz der Pertussis-spezifischen Impfantwort besitzt.

Die in Lancet Infectious Diseases publizierte Arbeit beschreibt die Ergebnisse einer Studie zur Sicherheit und Immunogenität eines rekombinanten azellulären PertussisImpfstoffs, der genetisch inaktiviertes Pertussistoxin und Filamentöses Hämagglutinin enthält, entweder als monovalenter Impfstoff aP (PTgen/FHA) oder in Kombination mit Tetanus und reduzierten Diphtherie-Impfkomponenten (TdaP/PTgen/ FHA), im Vergleich zu einem zugelassenen dTap Kombinationsimpfstoff.

Der neue TdaP (PTgen/FHA)-Impfstoff induziert 28 Tage nach der Impfung eine höhere Pertussis-Impfantwort als der zugelassene dTap-Boosterimpfstoff. Aufgrund der Studienergebnisse ist der neue azelluläre Pertussis-Impfstoff mit gentechnisch inaktiviertem Pertussis-Toxin in Thailand zwischenzeitlich zugelassen worden. Ob der neue Impfstoff eine höhere Persistenz des Pertussis-Impfschutzes erreicht, muss in weiteren Studien untersucht werden.

\section{Neuer Rotavirus-Impfstoff für Neugeborene}

Ein neuer, in Indonesien entwickelter Impfstoff gegen Rotavirus-Infektionen, der bereits wenige Tage nach der Geburt verabreicht werden kann, hat in einer randomisierten Studie [7] eine gute Schutzwirkung erzielt.

Nach Schätzungen der Weltgesundheitsorganisation starben im Jahr 2013 weltweit 215000 Kinder an Durchfallerkrankungen durch Rotaviren (RV). Die meisten Todesfälle hätten jedoch durch eine Impfung verhindert werden können. Nach Angaben der WHO haben unverändert etwa 90 Millionen Kinder in Ländern, in denen 90\% der RV-assoziierten Todesfälle auftreten, keinen Zugriff auf die derzeit verfügbaren Impfstoffe. Die Bill \& Melinda Gates Foundation hat deshalb die Entwicklung eines neuen Impfstoffes (RV3-BB) unterstützt, der gegenüber den beiden in den industrialisierten Ländern zugelassenen Impfstoffen den Vorteil hat, dass er direkt nach der Geburt verimpft werden kann, während die zugelassenen Impfstoffe erst ab einem Alter von 6 Wochen eingesetzt werden können. Gerade in nicht-industrialisierten Ländern mit begrenzten Möglichkeiten der Gesundheitsversorgung kann eine neonatale Impfung von Vorteil sein, um zuverlässig möglichst alle Säuglinge zu impfen und auch jene Kinder zu schützen, die in den ersten Lebenswochen erkranken.

Wie die schon zugelassenen Impfstoffe ist der neue Impfstoff RV3-BB ein Lebendimpfstoff. Nachdem RV3-BB sich in einer Phase-Il-Studie bei Neugeborenen und Säuglingen als sicher erwiesen hat, wurde er zwischen 2013 und 2016 an 1513 Neugeborenen und Säuglingen in Krankenhäusern und Einrichtungen der medizinischen Primärversorgung in Zentral-Java und Yogyakarta Indonesien getestet.

Schwere Rotavirus-Gastroenteritiden traten bis zum Alter von 18 Monaten bei 1,4\% der direkt nach der Geburt geimpften Kinder (7 von 498), bei 2,7\% der im späteren Säuglingsalter geimpften Kinder (14 von 511 ) und bei $5,6 \%$ in der Placebo-Gruppe (28 von 504 Babys) auf. Die Autoren ermitteln für den Impfbeginn nach Geburt eine Wirksamkeit der Vakzine gegen schwere Rotavirus-Gastroenteritis von 75\% (95\% KI: 44-91; $<<0,001)$ mit 18 Monaten und sogar $94 \%$ (95\% Kl: 56-99; $\mathrm{p}=0,006) \mathrm{mit}$ 12 Monaten.

Die nach Rotavirus-Impfungen gefürchtete Invagination wurde nur einmal beobachtet, wobei diese erst 114 Tage nach der letzten Impfung auftrat und wahrscheinlich nicht durch die Impfung bedingt war. Es handelt sich um eine interessante Impfstoffentwicklung, die insbesondere für Länder mit höherem Infektionsrisiko, wahrscheinlich auch assoziiert mit zum Teil ungünstigeren Lebensbedingungen und unterschiedlichen Hygienestandards, wichtig ist.

\section{Schutz vor RSV durch Imp- fung in der Schwangerschaft}

Respiratory-Syncytial-Virus (RSV) ist der häufigste Erreger von Atemwegsinfektionen im Säuglings- und Kleinkindalter. Aufgrund der ausgeprägten proinflammatorischen Abwehrreaktion in den ersten Le- 
bensjahren entwickeln Säuglinge und Kleinkinder das typische Krankheitsbild der Bronchiolitis. Gerade sehr junge Säuglinge und ehemalige Frühgeborene müssen wegen schwer verlaufender RSV-Infektionen hospitalisiert werden. Bisher steht nur eine passive Immunisierung mit einem humanisierten monoklonalen Antikörper als präventive Maßnahme zur Verfügung. Frühere Versuche zur Entwicklung eines aktiven Impfstoffes waren nicht erfolgreich und in der Vergangenheit mit schweren Komplikationen und Todesfällen belastet.

Die Arbeitsgruppe von Allison August arbeitet an der Entwicklung eines RSV-Fusion-Protein-Nanopartikel-Impfstoffs (RSVF-Impfstoff) zur Immunisierung von Schwangeren im dritten Trimenon, mit dem Ziel, Säuglinge passiv durch Übertragung von RSV-spezifischen maternalen Antikörpern zu schützen. In Vaccine [8] berichten die Forscher über die Ergebnisse einer Studie zur Immunogenität und Sicherheit verschiedener Formulierungen des RSV-F-Impfstoffes nach ein- oder zweimaliger Impfung.

Gesunde Frauen im Alter von 18 bis 35 Jahren erhielten intramuskulär an Tag 0 und 28 entweder Placebo oder RSV-F-Impfstoff adjuvantiert mit Aluminium. Die Immunogenität der Impfung wurde von Tag 0 bis Tag 91 durch Messung von Anti-F-IgG und Palivizumab-kompetitivem Antikörper (PCA) mittels ELISA sowie RSV-A und -B neutralisierenden Antikörpern mittels $\mathrm{Mi}$ kroneutralisations-Assay (MN) ermittelt. Alle in der Studie eingesetzten Formulierungen waren gut verträglich. Die AntiF-IgG- und PCA-Antworten zeigten nach beiden Impfungen einen deutlichen Anstieg, während der MN erst nach der ersten Dosis signifikant anstieg, dann aber ein Plateau entwickelte. Mittels WesternBlot konnte gezeigt werden, dass das Risiko für RSV-Infektionen bei aktiv Geimpften über den gesamten Beobach- tungszeitraum um 52\% ( $p=0,009$ insgesamt) reduziert war. Die Impf-Antwort erreicht in 14 Tagen eine maximale Antikörperantwort und persistiert über 91 Tage.

Die Entwicklung des RSV-FusionsproteinNanopartikel-Impfstoffs für Mütter ermöglicht den passiven Schutz von Neugeborenen und jungen Säuglingen gegenüber frühen RSV-Infektionen.

\section{Interessenkonflikte}

Markus Knuf: LKP und PI bei Impfstudien. Beratertätigkeit für GSK, Pfizer, Sanofi, MSD, SPMSD, Baxter, Novartis, AstraZeneca, Medimmune u.a. Präsentationen während Industrie-Symposien. Die o.g. Tätigkeiten nimmt der Autor als Dienstaufgabe wahr. Er erhält persönlich keine Honorare von Firmen. Es besteht diesbezüglich auch keine Zielvereinbarung mit seinem Dienstherren. Fred Zepp served as independent Chairman of the DSMB (Data Safety Moniotoring Board) during the clinical development of Sanofi Pasteur's quadrivalent Influenza Vaccine with infants and toddlers.

\section{Autoren}

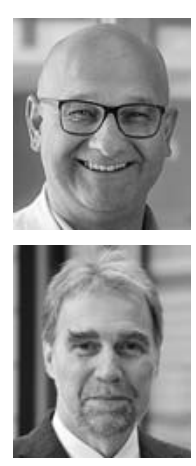

Markus Knuf ${ }^{1}$, Fred Zepp ${ }^{2}$

1 HELIOS Dr. Horst-SchmidtKliniken, Klinik für Kinder und Jugendliche

2 Universitätsmedizin der Johannes Gutenberg-Universität Mainz, Zentrum für Kinder- und Jugendmedizin

\section{Korrespondenzadresse}

Univ.-Prof. Dr. med. Markus Knuf HELIOS Dr. Horst-Schmidt-Kliniken Klinik für Kinder und Jugendliche Ludwig-Erhard-Str. 100 65199 Wiesbaden markus.knuf@helios-gesundheit.de
Univ.-Prof. Dr. med. Fred Zepp

Universitätsmedizin der Johannes Gutenberg-Universität Mainz

Zentrum für Kinder- und Jugendmedizin Langenbeckstraße 1

55131 Mainz

fred.zepp@unimedizin-mainz.de

\section{Literatur}

[1] Gandhi A, Balmer P, York LJ. Characteristics of a new meningococcal serogroup $B$ vaccine, bivalent rLP2086 (MenB-FHbp; Trumenba ${ }^{\circledR}$ ). Postgrad Med 2016; 128: 548556

[2] Ostergaard L, Vesikari T, Absalon J et al. A Bivalent Meningococcal B Vaccine in Adolescents and Young Adults. N Engl J Med 2017; 377: 2349-2362

[3] AG Influenza der Ständigen Impfkommission (STIKO). Wissenschaftliche Begründung für die Empfehlung des quadrivalenten saisonalen Influenzaimpfstoffs. Epid Bull 2018; 2: 19-31. doi:10.17886/EpiBull-2018-002

[4] Claeys C, Zaman K, Dhaibo G et al. Prevention of vaccine-matched and mismatched influenza in children aged 6-35 months: a multinational randomised trial across five influenza seasons. Lancet Child Adolec Health 2018; 2:338-349

[5] Pepin S, Dupuy M, Borja C et al. Intramuscular Quadrivalent Influenza Vaccine is Efficacious in Naive Children Aged 6 to 35 Months: a Large-Scale, Placebo-Controlled Trial. Poster ESP17-1082: 35th ESPID Meeting Mai 2327, 2017 - Madrid, Spanien. Im Internet: https://espid2017.kenes.com/Documents/ ESPID17\%20abstracts.pdf; Stand: 02.10.2018

[6] Sricharoenchai S, Sirivichayakul C, Chokephaibulkit $\mathrm{K}$ et al. A genetically inactivated two-component acellular pertussis vaccine, alone or combined with tetanus and reduced-dose diphtheria vaccines, in adolescents: a phase $2 / 3$, randomised controlled non-inferiority trial. Lancet Infect Dis 2018; 18: $58-67$

[7] Bines JE, At Thobari J, Satria CD et al. Human Neonatal Rotavirus Vaccine (RV3-BB) to Target Rotavirus from Birth. N Engl J Med 2018; 378: $719-730$

[8] August A, Glenn GM, Kpamegan E et al. A Phase 2 randomized, observer-blind, placebo-controlled, dose-ranging trial of aluminum-adjuvanted respiratory syncytial virus $\mathrm{F}$ particle vaccine formulations in healthy women of childbearing age. Vaccine 2017; 35: $3749-3759$ 